PROCEEDINGS OF THE

AMERICAN MATHEMATICAL SOCIETY

Volume 134, Number 12, December 2006, Pages 3629-3635

S 0002-9939(06)08483-8

Article electronically published on May 31, 2006

\title{
A LOWER BOUND FOR THE GROUND STATE ENERGY OF A SCHRÖDINGER OPERATOR ON A LOOP
}

\author{
HELMUT LINDE
}

(Communicated by Mikhail Shubin)

\begin{abstract}
Consider a one-dimensional quantum mechanical particle described by the Schrödinger equation on a closed curve of length $2 \pi$. Assume that the potential is given by the square of the curve's curvature. We show that in this case the energy of the particle cannot be lower than 0.6085 . We also prove that it is not lower than 1 (the conjectured optimal lower bound) for a certain class of closed curves that have an additional geometrical property.
\end{abstract}

\section{INTRODUCTION}

Let $\Gamma$ be a smooth closed curve of length $2 \pi$ in the plane with the curvature $\kappa(s)$ which is regarded as a function of the arc length. We consider the Schrödinger operator

$$
H_{\Gamma}=-\Delta+\kappa^{2}(s) \quad \text { in } \quad L^{2}([0,2 \pi))
$$

with periodic boundary conditions. Let $\lambda_{\Gamma}$ be the lowest eigenvalue of $H_{\Gamma}$. It has been conjectured that $\lambda_{\Gamma} \geq 1$ for any $\Gamma$. The class $F$ of the conjectured minimizers of $\lambda_{\Gamma}$ contains the circle and certain point-symmetric oval loops. For all curves in $F$ the equality $\lambda_{\Gamma}=1$ holds, but so far it has not been shown that this is actually the smallest possible value of $\lambda_{\Gamma}$. In their paper [1] Benguria and Loss established a connection between this problem and the Lieb-Thirring conjecture in one dimension. They also proved that $\lambda_{\Gamma} \geq 0.5$, which seems to be the best lower bound for $\lambda_{\Gamma}$ so far.

Recently, Burchard and Thomas have shown 2 that the curves in $F$ minimize $\lambda_{\Gamma}$ at least locally, i.e., there is no small variation around these curves that reduces $\lambda_{\Gamma}$. In the present article we will add further credibility to the mentioned conjecture in two ways. On the one hand, we show that $\lambda_{\Gamma} \geq 1$ holds for a considerable class of curves that meet a certain additional geometrical condition. Extending this method to the class of all curves of interest yields, on the other hand, an improved lower bound on $\lambda_{\Gamma}$.

\section{Statement of the Result}

For a given smooth curve $\Gamma$ with an arc length parameter $s$, we introduce the angle $\phi(s)$ between the tangent on $\Gamma$ in $s$ and some fixed axis, which implies $\phi^{\prime}(s)=$

Received by the editors June 21, 2005.

2000 Mathematics Subject Classification. Primary 81Q10; Secondary 53A04.

This work was supported by DIPUC (Pontificia Universidad Católica de Chile).

(C)2006 American Mathematical Society 
$\kappa(s)$. For the sake of simplicity we will only consider strictly convex curves, i.e., $\phi^{\prime}>0$. To keep the notation compact we write

$$
\phi: \Omega \rightarrow \Omega \text { with } \Omega:=\mathbb{R} / 2 \pi \mathbb{Z},
$$

considering numbers that differ by an integer multiple of $2 \pi$ as identical. Our main result is:

Theorem 2.1. Let $\Gamma$ be a smooth, strictly convex, closed curve of length $2 \pi$ in the plane and $\lambda_{\Gamma}$ defined as above. Then

$$
\lambda_{\Gamma}>\left(1+\frac{1}{1+8 / \pi}\right)^{-2}>0.6085 .
$$

In the proof of Theorem 2.1 we will make use of the following geometrical concept: We call $s \in \Omega$ a 'critical point' of $\Gamma$ if $\phi(s+\pi)=\phi(s)+\pi$. Obviously, $s+\pi$ is then also a critical point. If $s$ is a critical point, we call $\phi(s)$ a 'critical angle'. While open curves may have no critical points at all, the following lemma holds for the closed curves we are considering.

Lemma 2.2. Every smooth closed curve $\Gamma$ has at least six critical points.

It is clear from the definition of a critical point and the lemma that every $\Gamma$ has at least three critical points and three critical angles in $[s, s+\pi) \subset \Omega$ for any $s \in \Omega$. For a class of curves that have their critical angles distributed somewhat evenly, we can show that $\lambda_{\Gamma} \geq 1$ holds:

Theorem 2.3. Let $\Gamma$ be as in Theorem 2.1 and assume additionally that every interval $\left[\phi, \phi+\frac{\pi}{2}\right) \subset \Omega$ contains at least one critical angle of $\Gamma$. Then $\lambda_{\Gamma} \geq 1$.

It is an immediate consequence of Theorem 2.3 and Lemma 2.2 that for any hypothetical curve $\Gamma$ with $\lambda_{\Gamma}<1$ there is a $\phi$ such that $\left[\phi, \phi+\frac{\pi}{2}\right)$ and $\left[\phi+\pi, \phi+\frac{3 \pi}{2}\right)$ each contain at least three critical angles and $\left[\phi+\frac{\pi}{2}, s+\pi\right) \cup\left[\phi+\frac{3 \pi}{2}, \phi+2 \pi\right)$ none. A few comments on the geometrical interpretation of the above are in order: Although we have defined the critical points for a certain parameterization of the curve, the location of the critical points is an intrinsic property of the curve. More precisely, two points $P_{1}$ and $P_{2}$ on a closed curve of length $2 \pi$ are critical, if the arc length between $P_{1}$ and $P_{2}$ is $\pi$ and the tangents in these two points are parallel.

From Theorem 2.3 it follows that only curves with a rather uneven distribution of their critical points are candidates for $\lambda_{\Gamma}<1$. Roughly speaking, such curves tend to be rather symmetric, as will become clear in the proof of Theorem 2.1. In fact, the function $f$, that we will define and estimate in the proof, can in some sense be seen as a measure for how far away $\Gamma$ is from being point-symmetric. This, on the other hand, will enable us to estimate how far $\lambda_{\Gamma}$ could be below one.

We are not aware of any direct correlation between the distribution of the critical points and the ground state energy $\lambda_{\Gamma}$, except the connection that is established by Theorem 2.3. of course. There seems to be no reason why the condition of Theorem 2.3 should 'prefer' curves with a high energy, especially if one takes into account that the conjectured minimizers meet this condition. We believe that this makes the conjecture $\lambda_{\Gamma} \geq 1$ even more credible.

The remainder of the article is devoted to proving Lemma 2.2 and the two theorems. 


\section{Proof of the Results}

To prepare the proofs of Lemma 2.2 and the two theorems we introduce some more notation: We consider a curve $\Gamma$ as in Theorem 2.1 and assume without loss of generality that $\phi^{\prime}>0$. Because $\Gamma$ is closed, $\phi$ meets the conditions

$$
\begin{aligned}
& \int_{\Omega} \cos \phi(s) \mathrm{d} s=\int_{\Omega} \sin \phi(s) \mathrm{d} s=0, \\
& \int_{\Omega} \phi^{\prime}(s) \mathrm{d} s=2 \pi .
\end{aligned}
$$

We note that $\phi(s)$ has an inverse function $\phi^{-1}: \Omega \rightarrow \Omega$, and the closure conditions are equivalent to

$$
\begin{aligned}
& \int_{\Omega}\left(\phi^{-1}\right)^{\prime}(t) \sin t \mathrm{~d} t=\int_{\Omega}\left(\phi^{-1}\right)^{\prime}(t) \cos t \mathrm{~d} t=0, \\
& \int_{\Omega}\left(\phi^{-1}\right)^{\prime}(t) \mathrm{d} t=2 \pi .
\end{aligned}
$$

The function $\left(\phi^{-1}\right)^{\prime}$ can therefore be written as a Fourier series

$$
\left(\phi^{-1}\right)^{\prime}(t)=1+\sum_{n=2}^{\infty} n a_{n} \cos n t-n b_{n} \sin n t,
$$

such that

$$
\phi^{-1}(t)=C+t+\sum_{n=2}^{\infty} a_{n} \sin n t+b_{n} \cos n t
$$

By the invariance of the problem under a shift of the arc length parameter $s$, we can assume that $C=0$. Then we can write $\phi^{-1}$ in the form

$$
\phi^{-1}(t)=t+g(t)+f(t)
$$

where

$$
\begin{aligned}
g(t) & :=\sum_{n=2,4,6, \ldots}^{\infty} a_{n} \sin n t+b_{n} \cos n t, \\
f(t) & :=\sum_{n=3,5,7, \ldots}^{\infty} a_{n} \sin n t+b_{n} \cos n t .
\end{aligned}
$$

Note that

$$
f(t+\pi)=-f(t), \quad g(t+\pi)=g(t) \quad \text { for all } \quad t \in \Omega .
$$

Proof of Lemma 2.2. From (3.1) and (3.2) it is easy to see that the critical angles of $\Gamma$ are just the zeroes of $f$. By continuity of $f$ and (3.2), any nontrivial $f$ clearly has at least two zeroes $t_{0}$ and $t_{0}+\pi$ in $\Omega$ with a change of sign. But if these were the only zeroes, we would have

$$
\int_{\Omega} f(t) \sin \left(t-t_{0}\right) \mathrm{d} t \neq 0
$$

which is impossible by the definition of $f$. So $f$ must change its sign in at least one more point. By the symmetry property (3.2) it is clear that if, say, $f\left(t_{0}+\epsilon\right)>0$, then $f\left(t_{0}+\pi-\epsilon\right)>0$ for small $\epsilon>0$. That means that each of the intervals $\left(t_{0}, t_{0}+\pi\right)$ and $\left(t_{0}+\pi, t_{0}+2 \pi\right)$ contains an even number of zeroes with a change of sign. In total, this leads to a minimum of six zeroes of $f$ with a change of sign. 
We now state and prove a lemma that is key to the proofs of Theorem 2.1 and Theorem 2.3 ,

Lemma 3.1. Let $\Gamma$ be as in Theorem 2.1 and let $\left\{t_{i}\right\}_{i=1, \ldots, n} \subset \Omega$ be a set of numbers such that $\left[t, t+\frac{\pi}{2}\right] \cap\left\{t_{i}\right\} \neq \emptyset$ for all $t \in \Omega$. Assume that $\left|f\left(t_{i}\right)\right| \leq \alpha$ for all $i$. Then

$$
\lambda_{\Gamma} \geq(1+2 \alpha / \pi)^{-2} .
$$

Proof. Comparing (3.2) with (3.1) we see that

$$
\phi^{-1}(t+\pi)=\phi^{-1}(t)+\pi-2 f(t) \quad \text { for all } t \in \Omega .
$$

Now assume $R(s)>0$ to be the ground state of $H_{\Gamma}$ and define the functions

$$
x(s):=R(s) \cos \phi(s), \quad y(s):=R(s) \sin \phi(s) .
$$

Interpreted as Euclidean coordinates, $x$ and $y$ define a closed curve in the plane. In these coordinates the lowest eigenvalue $\lambda_{\Gamma}$ of $H_{\Gamma}$ is

$$
\lambda_{\Gamma}=\frac{\int_{\Omega}\left(R^{\prime}(s)^{2}+\phi^{\prime}(s)^{2} R(s)^{2}\right) \mathrm{d} s}{\int_{\Omega} R(s)^{2} \mathrm{~d} s}=\frac{\int_{\Omega}\left(x^{\prime}(s)^{2}+y^{\prime}(s)^{2}\right) \mathrm{d} s}{\int_{\Omega}\left(x(s)^{2}+y(s)^{2}\right) \mathrm{d} s} .
$$

We now define the orthogonal projections of the curve $(x(s), y(s))$ onto straight lines through the origin:

$$
h_{\beta}(s):=\left(\begin{array}{c}
\sin \beta \\
-\cos \beta
\end{array}\right) \cdot\left(\begin{array}{l}
x(s) \\
y(s)
\end{array}\right)=x(s) \sin \beta-y(s) \cos \beta .
$$

We note that

$$
h_{\beta}\left(\phi^{-1}(\beta)\right)=0
$$

and, by (3.3),

$$
h_{\beta}\left(\phi^{-1}(\beta)+\pi-2 f(\beta)\right)=h_{\beta}\left(\phi^{-1}(\beta+\pi)\right)=0 .
$$

This means that the quantity

$$
I(\beta):=\frac{\int_{\Omega} h_{\beta}^{\prime}(s)^{2} \mathrm{~d} s}{\int_{\Omega} h_{\beta}(s)^{2} \mathrm{~d} s},
$$

which is the Rayleigh-Ritz quotient for the Laplacian on $\Omega$ with Dirichlet conditions at $\phi^{-1}(\beta)$ and $\phi^{-1}(\beta)+\pi-2 f(\beta)$, can be estimated from below by

$$
I(\beta) \geq\left(1+\frac{2|f(\beta)|}{\pi}\right)^{-2} .
$$

Now we consider two cases: First, assume that there is no $\beta_{0}$ for which $I\left(\beta_{0}\right)=$ $(1+2 \alpha / \pi)^{-2}$. It is clear that $I(\beta)=1$ if $\beta$ is a zero of $f$ and we know that such a zero exists. By continuity of $I(\beta)$ in $\beta$ we conclude that in this case $I(\beta)>$ $(1+2 \alpha / \pi)^{-2}$ for all $\beta \in \Omega$. Choosing first $\beta=\pi / 2$ and then $\beta=0$ yields

$$
\frac{\int_{\Omega} x^{\prime 2} \mathrm{~d} s}{\int_{\Omega} x^{2} \mathrm{~d} s} \geq(1+2 \alpha / \pi)^{-2} \quad \text { and } \quad \frac{\int_{\Omega} y^{\prime 2} \mathrm{~d} s}{\int_{\Omega} y^{2} \mathrm{~d} s} \geq(1+2 \alpha / \pi)^{-2},
$$

such that $\lambda_{\Gamma} \geq(1+2 \alpha / \pi)^{-2}$ by (3.4). 
In the second case there is a $\beta_{0}$ with $I\left(\beta_{0}\right)=(1+2 \alpha / \pi)^{-2}$, and by rotational symmetry of the problem we can assume that $\beta_{0}=0$, i.e.,

$$
I(0)=\frac{\int_{\Omega} y^{\prime 2} \mathrm{~d} s}{\int_{\Omega} y^{2} \mathrm{~d} s}=(1+2 \alpha / \pi)^{-2} .
$$

Now put (3.5) and (3.6) into (3.7) and set $\beta=t_{i}$ to get

$$
\int_{\Omega}\left(-x^{\prime} \sin t_{i}+y^{\prime} \cos t_{i}\right)^{2} \mathrm{~d} s \geq(1+2 \alpha / \pi)^{-2} \int_{\Omega}\left(-x \sin t_{i}+y \cos t_{i}\right)^{2} \mathrm{~d} s .
$$

Using (3.8) this becomes

$$
\int_{\Omega}{x^{\prime}}^{2} \mathrm{~d} s \geq(1+2 \alpha / \pi)^{-2}\left(\int_{\Omega} x^{2} \mathrm{~d} s+\frac{2}{\tan t_{i}} \int_{\Omega}\left(x^{\prime} y^{\prime}-x y\right) \mathrm{d} s\right) .
$$

Because of the conditions on the distribution of the $t_{i}$ in $\Omega$ we can choose $i$ such that the second summand in the bracket on the right side of (3.9) is positive. Thus,

$$
\int_{\Omega}{x^{\prime 2}}^{2} \mathrm{~d} \geq(1+2 \alpha / \pi)^{-2} \int_{\Omega} x^{2} \mathrm{~d} s .
$$

Lemma 3.1 now follows from the combination of (3.8) with (3.10).

Proof of Theorem 2.3. Let $\left\{t_{i}\right\}_{i=1, \ldots, n} \subset \Omega$ be the set of critical angles of $\Gamma$. Then by the assumption of Theorem 2.3 this set also meets the conditions of Lemma 3.1 Being critical angles, the $t_{i}$ 's satisfy $f\left(t_{i}\right)=0$. Thus $\alpha$ in Lemma 3.1 can be chosen to be zero, and Theorem 2.3 follows.

Proof of Theorem 2.1. To prove Theorem 2.1 we will derive estimates on the function $f(t)$ as defined in (3.1) and then apply Lemma 3.1. It is obvious that we only have to consider curves that are not covered by Theorem 2.3 This means that our $\Gamma$ has an interval larger than $\frac{\pi}{2}$ without critical angles. Recall that the critical angles of $\Gamma$ are just the zeroes of $f$. We will thus assume, without losing generality, that $t_{0}$ (with $0<t_{0}<\frac{\pi}{2}$ ) and $\pi$ are zeroes of $f$ with a change of sign and that $f(t)>0$ for $t \in\left(t_{0}, \pi\right)$. We define $\Omega_{0}:=\left[t_{0}, \pi\right]$. Let $\Omega_{+}$and $\Omega_{-}$be the sets of all points $t \in\left[0, t_{0}\right)$ where $f(t)$ is positive or negative, respectively. Let us now collect some information on $f$ :

First, we show that

$$
\int_{\Omega}\left|f^{\prime}(t)\right| \mathrm{d} t \leq 2 \pi .
$$

To do so, we note that $\left(\phi^{-1}\right)^{\prime}>0$ because we assumed $\phi^{\prime}>0$ earlier. By (3.1) this means

$$
f^{\prime}(t)+g^{\prime}(t)>-1 \quad \text { for all } t \in \Omega .
$$

But, applying (3.2) to this inequality, we also get

$$
-f^{\prime}(t)+g^{\prime}(t)>-1 \quad \text { for all } t \in \Omega .
$$

Putting together the last two inequalities, we get

$$
\left|f^{\prime}(t)\right|<1-g^{\prime}(t) \quad \text { for all } t \in \Omega .
$$

Integrating over $\Omega$ and keeping in mind the periodicity of $g$ yields (3.11). 
Second, we note that for any $\Delta, t_{0} \in \Omega$

$$
\begin{aligned}
0 & =\int_{\Omega} f(t) \sin (t+\Delta) \mathrm{d} t \\
& =\int_{t_{0}}^{t_{0}+\pi} f(t) \sin (t+\Delta) \mathrm{d} t+\int_{t_{0}+\pi}^{t_{0}+2 \pi} f(t) \sin (t+\Delta) \mathrm{d} t \\
& =2 \int_{t_{0}}^{t_{0}+\pi} f(t) \sin (t+\Delta) \mathrm{d} t .
\end{aligned}
$$

Third, let us assume that there is an interval $\left[t_{1}, t_{1}+\frac{\pi}{2}\right] \subset \Omega_{0}$ with $f(t)>\alpha$ on $\left[t_{1}, t_{1}+\frac{\pi}{2}\right]$ for some $\alpha \in \mathbb{R}$. Then

$$
\int_{\Omega_{+}} f(t) \mathrm{d} t \geq \alpha \quad \text { and } \quad-\int_{\Omega_{-}} f(t) \mathrm{d} t \geq \alpha .
$$

This can be seen with the help of (3.12) via

$$
\begin{aligned}
0 & =\int_{0}^{\pi} f(t) \sin \left(t-t_{0}\right) \mathrm{d} t \\
& =\int_{\Omega_{+}} f(t) \sin \left(t-t_{0}\right) \mathrm{d} t+\int_{\Omega_{-}} f(t) \sin \left(t-t_{0}\right) \mathrm{d} t+\int_{\Omega_{0}} f(t) \sin \left(t-t_{0}\right) \mathrm{d} t \\
& \geq-\int_{\Omega_{+}} f(t) \mathrm{d} t+\alpha \int_{t_{1}}^{t_{1}+\pi / 2} \sin \left(t-t_{0}\right) \mathrm{d} t \\
& \geq-\int_{\Omega_{+}} f(t) \mathrm{d} t+\alpha \int_{0}^{\pi / 2} \sin (t) \mathrm{d} t \\
& =-\int_{\Omega_{+}} f(t) \mathrm{d} t+\alpha .
\end{aligned}
$$

The corresponding inequality for $\Omega_{-}$is proven analogously, exploiting once again (3.2).

Because $f$ vanishes at the edges of $\Omega_{0}$ and because $\max _{t \in \Omega_{0}} f(t)>\alpha$, it is clear that

$$
\int_{\Omega_{0}}\left|f^{\prime}(t)\right| \mathrm{d} t>2 \alpha
$$

From (3.13) we conclude that the inequalities

$$
\max _{t \in \Omega_{+}} f(t) \geq \frac{\alpha}{\left|\Omega_{+}\right|} \text {and } \max _{t \in \Omega_{-}}|f(t)| \geq \frac{\alpha}{\left|\Omega_{-}\right|}
$$

hold. Therefore

$$
\begin{aligned}
\int_{\Omega}\left|f^{\prime}(t)\right| \mathrm{d} t & =2\left(\int_{\Omega_{0}}\left|f^{\prime}(t)\right| \mathrm{d} t+\int_{\Omega_{+}}\left|f^{\prime}(t)\right| \mathrm{d} t+\int_{\Omega_{-}}\left|f^{\prime}(t)\right| \mathrm{d} t\right) \\
& \geq 2\left(2 \alpha+2 \frac{\alpha}{\left|\Omega_{+}\right|}+2 \frac{\alpha}{\left|\Omega_{-}\right|}\right) \\
& >4 \alpha\left(1+\frac{8}{\pi}\right) .
\end{aligned}
$$

In the last step we have used $\left|\Omega_{+}\right|+\left|\Omega_{-}\right|<\frac{\pi}{2}$. Comparing (3.14) with (3.11) shows that

$$
\alpha<\frac{\pi}{2(1+8 / \pi)} .
$$


We conclude that for any curve $\Gamma$ we can can find a sequence $\left\{t_{i}\right\}$ that meets the conditions of Lemma 3.1 for some $\alpha<\frac{\pi}{2(1+8 / \pi)}$, proving Theorem 2.1 ,

\section{ACKNowledgments}

It is a pleasure for me to express my gratitude to Professor Rafael D. Benguria for many helpful discussions and for proofreading the manuscript.

\section{REFERENCES}

1. R.D. Benguria and M. Loss: Connection between the Lieb-Thirring conjecture for Schrödinger operators and an isoperimetric problem for ovals in the plane, Contemporary Math. 362 (2004), 53-61. MR2091490(2005f:81057)

2. A. Burchard and L.E. Thomas: On an isoperimetric inequality for a Schrödinger operator depending on the curvature of a loop, Journal of Geometric Analysis 15 (2005), 543-563. MR 2203162

3. P. Exner, E.M. Harrell and M. Loss: Optimal eigenvalues for some Laplacians and Schrödinger operators depending on curvature, Mathematical results in quantum mechanics (Prague 1998), Oper. Theory Adv. Appl. 108 (1999), 47-58. MR.1708787 (2000e:58045)

4. E.M. Harrell: Gap estimates for Schrödinger operators depending on curvature, talk delivered at the 2002 UAB International Conference on Differential Equations and Mathematical Physics. Available electronically at http://www.math.gatech.edu/ ${ }^{\sim}$ harrell/

5. E.M. Harrell and M. Loss: On the Laplace operator penalized by mean curvature, Commun. Math. Phys. 195 (1998), 643-650. MR1641019 (99f:58213)

6. E.H. Lieb and W. Thirring: Inequalities for the moments of the eigenvalues of the Schrödinger Hamiltonian and their relation to Sobolev inequalities, Studies in Math. Phys., Essays in Honor of Valentine Bargmann, Princeton (1976), 269-303.

Department of Physics, Pontificia Universidad Católica de Chile, Casilla 306, Correo 22 Santiago, Chile

E-mail address: Helmut.Linde@gmx.de 